\title{
Anti-epidermal growth factor receptor monoclonal antibody-based therapy for metastatic colorectal cancer: a meta-analysis of the effect of PIK3CA mutations in KRAS wild-type patients
}

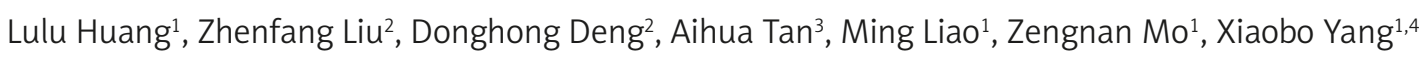

\author{
${ }^{1}$ Center for Genomic and Personalized Medicine, Guangxi Medical University, \\ Nanning, Guangxi, China \\ ${ }^{2}$ Hematology Department, the First Affiliated Hospital of Guangxi Medical University, \\ Nanning, Guangxi, China \\ ${ }^{3}$ Department of Chemotherapy, the Affiliated Tumor Hospital of Guangxi Medical \\ University, Nanning, Guangxi, China \\ ${ }^{4}$ Department of Occupational Health and Environmental Health, School of Public \\ Health, Guangxi Medical University, Nanning, Guangxi, China
}

Submitted: 27 February 2013

Accepted: 28 May 2013

Arch Med Sci 2014; 10, 1: 1-9

DOI: 10.5114/aoms.2014.40728

Copyright $\odot 2014$ Termedia \& Banach

\begin{abstract}
Introduction: We conducted a meta-analysis to dissect the association between PIK3CA mutations (exon 9 and exon 20) and resistance to anti-epidermal growth factor receptor (EGFR) monoclonal antibodies (MoAbs) in KRAS wild-type metastatic colorectal cancer (mCRC) patients.

Material and methods: In 11 previously published studies, 864 cancer patients were treated with cetuximab or panitumumab-based therapy. Primary outcomes included objective response (complete response + partial response vs. stable disease + progressive disease), progression-free survival (PFS), and overall survival (OS). We calculated the odds ratio (OR) or hazard ratio (HR) with $95 \%$ confidence intervals (Cls) to estimate the risk or hazard. We found consistent and clinically substantial risk or hazard for objective response, PFS, and OS in the cetuximab or panitumumab-treated mCRC patients.

Results: PIK3CA mutations as a whole were associated with reduced response and poor PFS and OS in KRAS wild-type $\mathrm{mCRC}$ patients (objective response: $\mathrm{OR}=0.42$ and $95 \% \mathrm{Cl} 0.23-0.75 ; \mathrm{PFS}: \mathrm{HR}=1.54$ and $95 \% \mathrm{Cl} 1.13-2.09$; and OS: $\mathrm{HR}=1.4$ and $95 \% \mathrm{Cl} 1.02-1.91)$. PIK3CA exon 9 mutations had no effect, whereas exon 20 mutations were associated with a worse outcome compared with wild types, with an OR of 0.21 (95\% Cl 0.05-0.93).

Conclusions: PIK3CA mutations as a whole might be useful prognostic factors for assessing clinical outcomes of anti-EGFR MoAb-based chemotherapies in KRAS wild-type mCRC patients. In particular, PIK3CA exon 20 mutations were significantly associated with lack of response.
\end{abstract}

Key words: colorectal cancer, PIK3CA, monoclonal antibodies, meta-analysis.

\section{Introduction}

Colorectal cancer is one of the most common cancers worldwide and one of the leading causes of cancer mortality. There has been an increase in the incidence of colorectal cancer in Poland [1]. Despite ad-

\author{
Corresponding author: \\ Prof. Xiaobo Yang \\ Center for Genomic \\ and Personalized Medicine \\ Guangxi Medical University \\ Shuangyong Road 22 \\ 530021 Nanning, China \\ Phone: 86-18677146179 \\ E-mail: 47628655@qq.com
}


vances in chemotherapy, the 5 -year relative survival remains poor at just $11 \%$ for patients with metastatic colorectal cancer (mCRC) [2]. Currently, two monoclonal antibodies (MoAbs) targeted at epidermal growth factor receptor (EGFR), the chimeric IgG1 MoAb cetuximab and the fully humanized IgG2 MoAb panitumumab, have shown a relevant clinical effect in treatment of patients with chemotherapy-refractory $\mathrm{MCRC}$ [3-6]. Because of common resistance to anti-EGFR MoAbs, recent guideline recommendations suggest that anti-EGFR MoAbs be given only to patients with KRAS wild-type mCRC [7, 8]. However, even for non-carriers of KRAS mutations, the response rate to anti-EGFR MoAbs is not high, ranging from $17 \%$ to $60 \%$, and only a subset of patients benefit from this treatment [9-18]. This heterogeneity suggests that there may be other predictive variables, besides KRAS, that determine responsiveness to anti-EGFR. Thus, the predictive value of additional mutations and deregulations of signaling pathways downstream of EGFR such as BRAF, PIK3CA or PTEN is currently under intensive investigation.

Specifically, what is highlighted in the explorations is PIK3CA. PIK3CA encodes the p110 $\alpha$ catalytic subunit of the class IA phosphatidylinositol 3-kinases (PI3Ks). Tumor-derived PIK3CA mutations lead to constitutive activation of p110 $\alpha$ enzymatic activity, stimulate the AKT pathway, and promote cell growth [19]. PIK3CA is frequently mutated in several malignancies such as colon, breast, brain, ovarian, liver, and lung cancers [20]. Mutation frequencies in CRC vary from $10 \%$ to $30 \%[17,21]$. The vast majority of activating PIK3CA mutations map to 3 sites: exon 9, codons 542 and 545 in the helical domain, and exon 20, codon 1047 in the kinase domain.

Emerging data have suggested that PIK3CA mutation is likely to be predictive of a lack of benefit from anti-EGFR therapy in $\mathrm{mCRC}$ [22-24], but the results are still inconclusive, partially due to the inclusion of molecularly unselected populations. For example, Prenen et al. suggested that PIK3CA mutations were not a major determinant of resistance to the anti-EGFR MoAbs in unselected mCRC patients [25], whereas De Roock et al. found that patients with PIK3CA mutations had a significantly lower response rate in KRAS wild-type patients [26]. Besides, the relatively small sample size of each study may have an effect. For example, Sartore-Bianchi et al. suggested that PIK3CA mutations were significantly associated with clinical resistance to anti-EGFR MoAbs [22] while Moroni et al. showed that PIK3CA alterations did not correlate with response [21]. Therefore, it is necessary to conduct a new meta-analysis to derive a more precise estimation of predictive value of PIK3CA mutations in KRAS wild-type $\mathrm{mCRC}$ patients treated with anti-EGFR MoAbs.

\section{Material and methods}

\section{Study selection}

Systematic computerized searches of the PubMed and HuGENet databases (up to $25^{\text {th }}$ February 2013) were performed. The following search terms were used: 'cetuximab', 'panitumumab', 'colon cancer', 'rectal cancer', 'colorectal cancer', 'colorectal neoplasm', 'CRC', 'PIK3CA', 'phosphoinositide-3-kinase catalytic', ' $\alpha$ polypeptide', 'PI 3-kinases'. References of the retrieved articles were further screened for earlier original studies. The inclusion criteria were as follows: (i) the studies focused on $\mathrm{mCRC}$ patients; (ii) those exploring the relation between PIK3CA mutations and clinical outcomes of KRAS wild-type $\mathrm{mCRC}$ patients treated with anti-EGFR MoAbs; (iii) those using one or more of the following as outcomes to assess tumor response and survival: objective response, PFS, and OS.

\section{Statistical analysis}

For each outcome measure, we estimated effects separately for patients with wild-type PIK3CA and PIK3CA mutations. For overall response rate, an odds ratio (OR) was calculated from the reported number of objective response (complete response $(C R)+$ (partial response $P R)$ ) and no response (progressive disease $(\mathrm{PD})+$ stable disease (SD)) in each arm, using the WHO criteria or RECIST (Response Evaluation Criteria in Solid Tumors) criteria [27]. In order to dissect the complicated relation between PIK3CA status and prognosis in relation to the treatment, we did stratified analyses and estimated the pooled OR according to KRAS mutation status. As different biological effects have been suggested for PIK3CA exon 9 (helical domain) and exon 20 (kinase domain) mutations [28-30], the OR or hazard ratio (HR) was also estimated for each type of mutation as well as for any PIK3CA mutations. Progression-free survival (PFS) and overall survival (OS) were evaluated by pooled Cox proportional HRs and $95 \% \mathrm{Cls}$ by published methods. The between-study heterogeneity was evaluated with $R^{2}$ and $50 \%$ in $R^{2}$ was regarded as the threshold. We carried out initial analyses with a fixed effect model and confirmatory analyses with a random effect model, if there was significant heterogeneity. We used inverted funnel plots and the Egger test to examine the effect of publication bias. All analyses were carried out using the Stata 9.2 and RevMan 5.0 software.

\section{Results}

The literature search (as of February 25, 2013) yielded 95 potentially assessable publications. Of these, 74 were excluded for the following reasons: not original studies, not human studies, not cohort 
studies and irrelevant studies. After reviewing the remaining 21 studies, we excluded 1 study which was not a retrospective cohort study [31], 1 study [22] that used the same data in another included study [9], 1 study in which no mutation in PIK3CA was found in any patient [32] and 7 studies which did not provide data in KRAS wild-type patients[14, 17, 33-37]. Finally, 11 relevant studies, comprising a total of 864 patients, were included. The flowchart of the reviews showed the detailed process of selection (Figure 1). The main characteristics of included studies are shown in Table I. Of the 11 studies, sample sizes ranged from 18 to 339. All of these studies were of retrospective design. Anti-EGFR MoAbs were given as second line or more in all the studies. The patients all received anti-EGFR MoAb-based treatment.

\section{PIK3CA mutations and objective response in KRAS wild-type patients}

Nine studies including 693 patients were eligible for the final analysis. PIK3CA mutations as a whole were associated with reduced objective response in KRAS wild-type $\mathrm{mCRC}$ patients $(\mathrm{OR}=0.42$;
95\% Cl 0.23-0.75; $p=0.003$; Figure 2). In stratified analyses, the association remained significant in subgroups of exon 20 mutations ( $O R=0.21$; 95\% Cl 0.05-0.93; $p=0.04)$, whereas exon 9 mutations had no effect $(\mathrm{OR}=0.54 ; 95 \% \mathrm{Cl} 0.26-1.12$; $p=0.10)$. Given that all the ORs for the exon 9 subgroup were less than 1 except the study of Moroni et al., we performed an influence analysis by excluding the study. The result was similar to that of the former $(\mathrm{OR}=0.46 ; 95 \% \mathrm{Cl} 0.21-1.01$; $p=0.05$; data not shown). No publication bias was detected by either the funnel plot or the Egger test ( $p=0.065, p=0.060, p=0.185$ for all exons, exon 20 and exon 9 , respectively).

\section{PIK3CA mutations and progression-free survival and overall survival in KRAS wild-type patients}

Four studies including 545 patients were eligible for the final analysis. The PIK3CA mutations were associated with a significant increase of hazard for PFS in all patients (mutant vs. wild-type: $\mathrm{HR}=1.54 ; 95 \% \mathrm{Cl} 1.13-2.09 ; p=0.006$; Figure 3). No significant between-study heterogeneity was

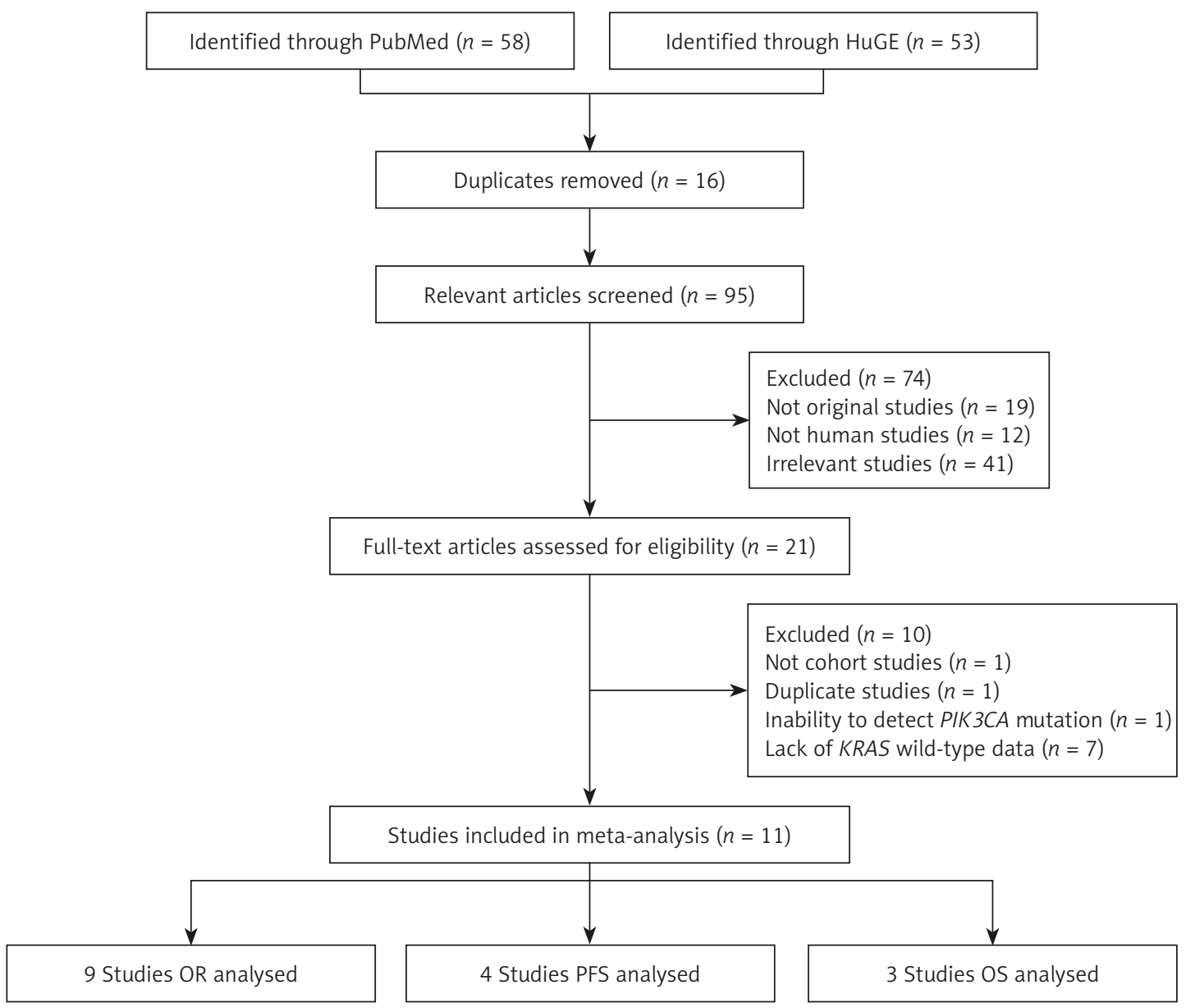

Figure 1. Flow chart for the process of selecting eligible publications 


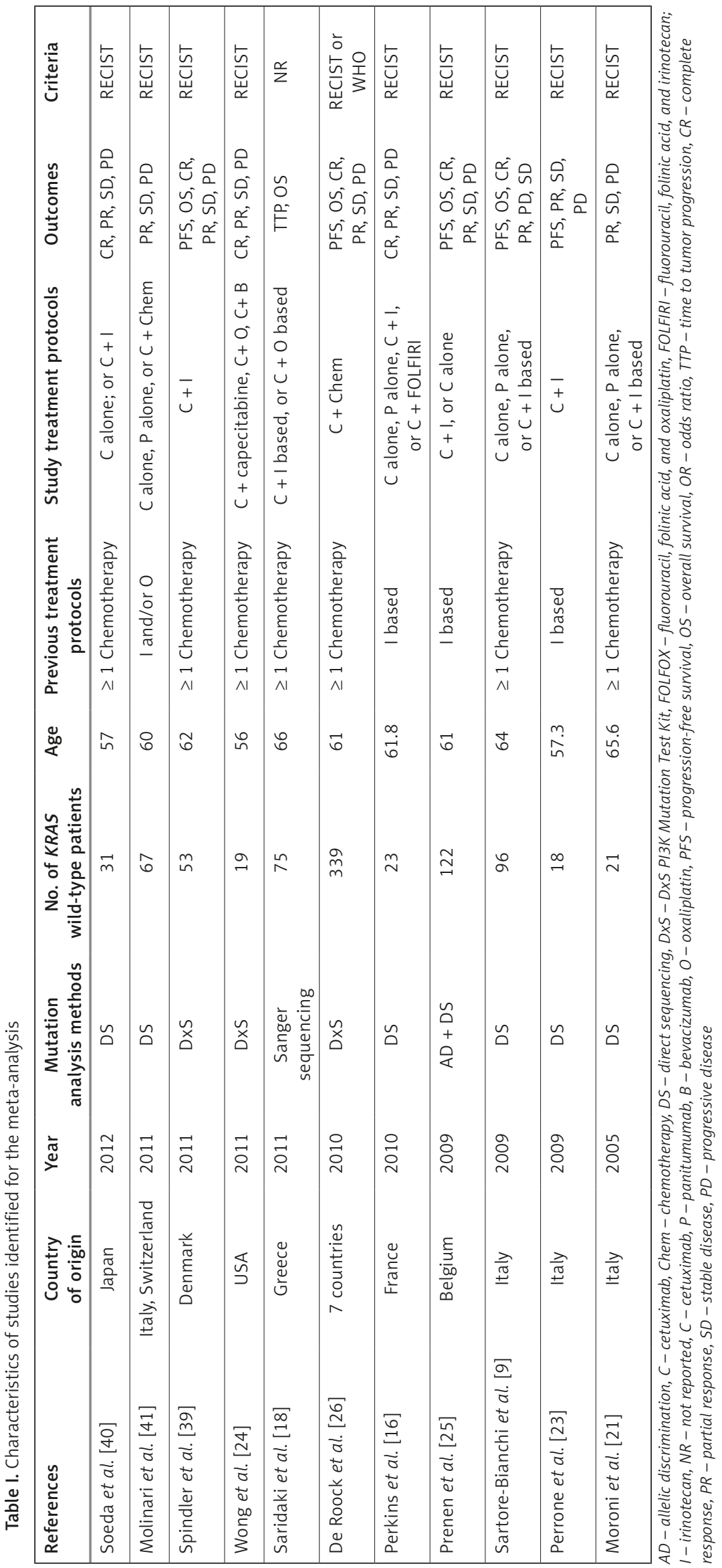




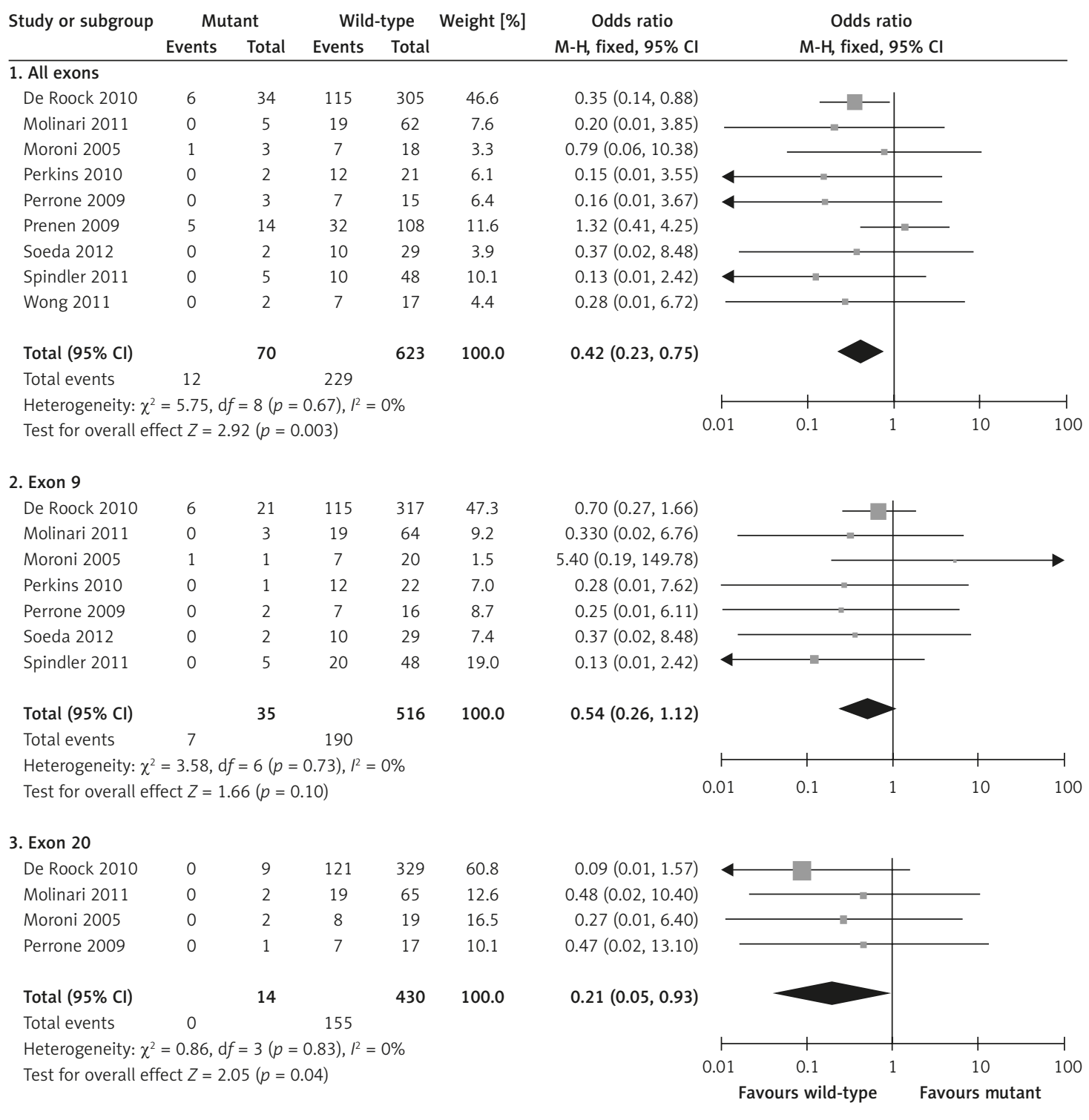

$\mathrm{Cl}$ - confidence interval, M-H - Mantel-Haenszel, Mutant - PIK3CA mutant, Wild-type - PIK3CA wild-type

Figure 2. Forest plot of the analysis of the relative risk according to the PIK3CA exon of the mutation in KRAS wildtype patients

found in the initial fixed model, so a random model did not need to be performed $\left(I^{2}=36.6 \%\right)$. Additionally, Egger's test did not show publication bias ( $p=0.894)$, and no significant outcome of influence analysis was observed (data are not shown).

Three studies including 527 patients were eligible for the final analysis. There was a substantial effect of PIK3CA mutations on death hazard in all patients (mutant vs. wild-type: $\mathrm{HR}=1.40$; $95 \% \mathrm{Cl} 1.02-1.91 ; p=0.036$; Figure 4). No significant between-study heterogeneity was observed in the initial fixed model $\left(R^{2}=0.0 \%\right)$. Moreover, no publication bias was detected with a $p$ value of 0.552 in Egger's test, and no significant outcome of influence analysis was observed (data are not shown).

\section{Discussion}

This meta-analysis used objective response, PFS, and OS as primary parameters to assess the influence of PIK3CA mutations on clinical outcomes of anti-EGFR MoAb chemotherapy in $\mathrm{mCRC}$ with KRAS wild-type. Our results imply that PIK3CA mutations were a biomarker of low objective response as well as short PFS and OS in KRAS wild-type patients with anti-EGFR MoAb chemotherapy.

Our study has some strengths although a similar meta-analysis was conducted before. The previous meta-analysis suggested that PIK3CA exon 20 mutations might be a potential biomarker for resistance to anti-EGFR MoAbs in KRAS wild-type $\mathrm{mCRC}$ [38], even though it failed to reach stati- 


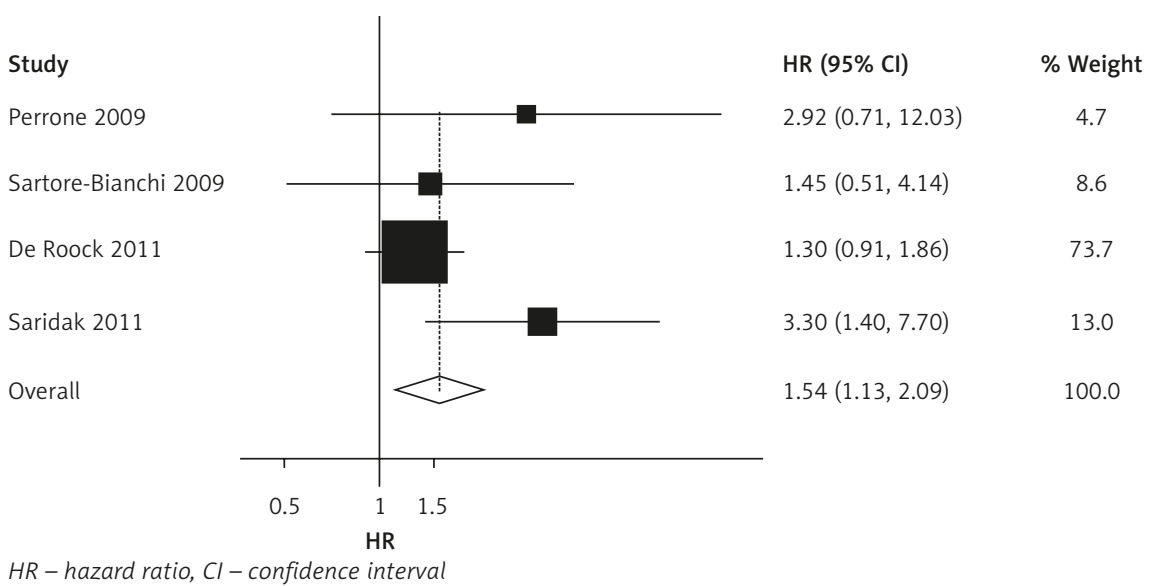

Figure 3. Forest plot of PFS according to the PIK3CA mutation in KRAS wild-type patients (mut vs. wt, reference group $=w t)$

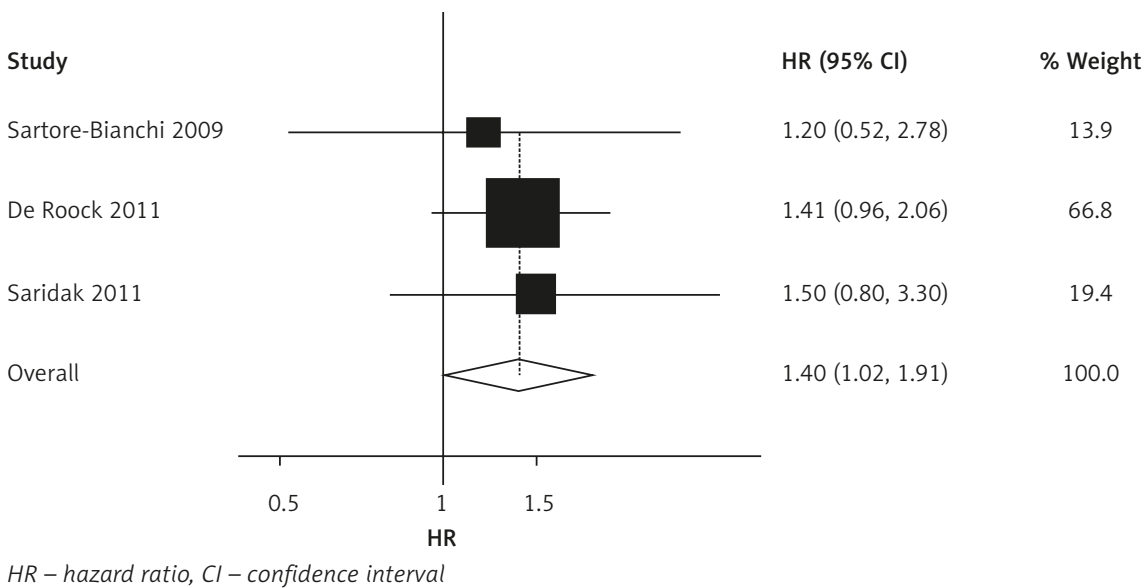

Figure 4. Forest plot of OS according to the PIK3CA mutation in KRAS wild-type patients (mut vs. wt, reference group $=\mathrm{wt}$ )

stical significance, most likely due to the small sample size. Moreover, flaws in the previous meta-analysis were that PFS and OS data were not sensibly combined. Because of the different ways and the incompleteness of reported data, the previous authors stated that the results could not be combined. But we believe that it is possible to combine the survival data if they were made full use of. For example, there were two duplicate studies, and we included a study [9] which provided survival data in wild-type KRAS patients, while the previous authors excluded it. In addition, a study was available because original data information was provided by the authors [23]. Recently, 6 studies with a larger sample size have been reported [32, 36, 37, 39-41]. Several studies have suggested that PIK3CA mutation was likely to be predictive of a lack of benefit from anti-EGFR therapy in $\mathrm{mCRC}$. Among them, two studies $[39,40]$ provided data in KRAS wild-type patients and two studies $[36,37]$ were in patients unselected by KRAS mutation status. However, one study suggested that PIK3CA alterations were not associated with response [41]. Considering the inconsistent conclusions, we updated the previous meta-analysis and confirmed its finding that PIK3CA exon 20 mutations were a potential biomarker for a low objective response in KRAS wildtype patients treated with anti-EGFR MoAbs.

Notably, the result is different if mutations on exon 9 and exon 20 are considered separately, as confirmation of the fact that the two exons have different mechanisms of action and related effects [42]. To induce transformation exon 20 mutants depend on binding with the regulatory subunit p85 $\alpha$, whereas exon 9 mutants circumvent p $85 \alpha$ binding but depend on RAS binding instead [43, 44]. Regarding objective response, exon 20 mutations alone significantly decrease the chance of achieving an objective response, whereas exon 9 mutations are unable to reproduce these data (Figure 2). Unfortunately, the survival results could not be divided into exon 9 and exon 20 mutations in a meta-analysis so far. Only one study by De Roock et al. [26] provided survival data according to the PIK3CA exon of the mutations. It reported that PIK3CA exon 20 mutations were statistically significantly associated with shorter PFS and OS 
in KRAS wild-type patients. It seems that the predictive power of exon 20 mutation is greater than that of any exon mutations and exon 9 mutations. A recent study in which stage $\chi$ CRC patients were present in 151 (13\%) cases found that coexistence of PIK3CA exon 9 and 20 mutations, but not PIK3CA mutation in either exon 9 or 20 alone, was associated with poor prognosis of CRC (not $\mathrm{mCRC}$ ) patients [45]. So far, it is not certain whether exon 20 mutation could be a potential biomarker for resistance to anti-EGFR MoAbs in KRAS wild-type $m C R C$. Taking lessons from studies in which the distinctive prognostic role of exon 9 and exon 20 mutations has been described, additional larger studies are still warranted to investigate the possibilities since the ultimate aim of the treatment of $\mathrm{mCRC}$ is to prolong the survival without affecting health-related quality of life parameters.

Despite our efforts to make an accurate and comprehensive analysis, limitations of our meta-analysis need to be addressed. First, most of the included studies were retrospective and not all the methods of PIK3CA mutation determination were consistent. This may have caused heterogeneity between studies. Second, not all treatment arms used anti-EGFR MoAbs as a single compound but in combination with irinotecan, oxaliplatin or 5-Fu, which could cause some bias in our estimates but was unlikely to change our major conclusions, because no studies showed that chemotherapy correlated with PIK3CA. Third, only 4 studies presented data on HR with $95 \% \mathrm{Cl}$ for PFS and only three studies presented data on $\mathrm{HR}$ with $95 \% \mathrm{Cl}$ for OS. The relatively small sample size might not have enough statistical power to detect the real association. Fourth, our analysis largely used unadjusted estimates, because not all published studies presented adjusted estimates, or when they did, the estimates were not adjusted by the same potential confounders. Fifth, we were unable to analyze the association between PIK3CA mutations and cetuximab or panitumumab toxicities, because few studies provided this information or used different toxicity profiles. Finally, our study was restricted to mCRC patients with KRAS wildtype, so we failed to assess the influence of other mutations. BRAF mutations have been associated with a poor prognosis in colorectal cancer [46] and might therefore confound analyses of PIK3CA mutations and survival in colorectal cancer. In contrast, Ulivi et al. concluded that BRAF and PIK3CA mutations would seem to be independent predictors of anti-EGFR therapy effectiveness [37]. PIK3CA exon 9 mutations in primary tumors and loss of PTEN nuclear expression in metastases correlated with KRAS mutations [47]. We were unable to investigate potential interactions between PIK3CA mutations on exon 9 and exon 20 due to the limited publications available on this topic in which only one sample carried a mutation in both exon 9 and exon 20 [25].

Overall, our meta-analysis showed that PIK3CA mutations as a whole might be useful prognostic factors for assessing clinical outcomes and further confirmed that PIK3CA mutation on exon 20 decreases the response rate of anti-EGFR MoAbbased chemotherapies in wild-type KRAS mCRC patients. But we could not exclude the potential confounding by the interaction effect of other mutations which frequently associated with PIK3CA exon 20 mutations. We also strongly recommend that exon 9 and 20 mutations be studied separately. And future prospective studies with a larger sample size are required to confirm our findings.

\section{Acknowledgments}

Lulu Huang and Zhenfang Liu have contributed equally to this work and should be considered as co-first authors.

This study was partially supported by grants from the National Natural Science Foundation of China (81060234, 81160072), Key Program and University Talents Highland Innovation Team of Guangxi(2012012D003,GJR201147-09), Chairman Science and Technology Fund and Tackle Program of Guangxi (1116-03, GKG1298003-07-01), Guangxi Science Fund for Distinguished Young Scholars (2012jjFA40011) and the Guangxi Natural Science Fund (2010GXNSFB013064, 2010GXNSFA013181).

\section{References}

1. Klimczak A, Kempinska-Miroslawska B, Mik M, Dziki L, Dziki A. Incidence of colorectal cancer in Poland in 1999-2008. Arch Med Sci 2011; 7: 673-78.

2. Jemal A, Siegel R, XU J, Ward E. Cancer statistics, 2010. CA Cancer J Clin 2010; 60: 277-300.

3. Saltz LB, Meropol NJ, Loehrer PJ Sr, Needle MN, Kopit J, Mayer RJ. Phase II trial of cetuximab in patients with refractory colorectal cancer that expresses the epidermal growth factor receptor. J Clin Oncol 2004; 22: 1201-8.

4. Cunningham D, Humblet $Y$, Siena $S$, et al. Cetuximab monotherapy and cetuximab plus irinotecan in irinotecan-refractory metastatic colorectal cancer. N Engl J Med 2004; 351: 337-45.

5. Lenz HJ, Van Cutsem E, Khambata-Ford S, et al. Multicenter phase II and translational study of cetuximab in metastatic colorectal carcinoma refractory to irinotecan, oxaliplatin, and fluoropyrimidines. J Clin Oncol 2006; 24: 4914-21.

6. Van Cutsem E, Peeters M, Siena S, et al. Open-label phase III trial of panitumumab plus best supportive care compared with best supportive care alone in patients with chemotherapy-refractory metastatic colorectal cancer. J Clin Oncol 2007; 25: 1658-64.

7. Allegra CJ, Jessup JM, Somerfield MR, et al. American Society of Clinical Oncology provisional clinical opinion: testing for KRAS gene mutations in patients with 
metastatic colorectal carcinoma to predict response to anti-epidermal growth factor receptor monoclonal antibody therapy. J Clin Oncol 2009; 27: 2091-6.

8. Morton RF, Hammond EH. ASCO Provisional Clinical Opinion: KRAS, cetuximab, and panitumumab-clinical implications in colorectal cancer. J Oncol Pract 2009; 5: 71-2.

9. Sartore-Bianchi A, Di Nicolantonio F, Nichelatti M, et al. Multi-determinants analysis of molecular alterations for predicting clinical benefit to EGFR-targeted monoclonal antibodies in colorectal cancer. PLoS One 2009; 4: e7287.

10. Di Fiore F, Blanchard F, Charbonnier F, et al. Clinical relevance of KRAS mutation detection in metastatic colorectal cancer treated by Cetuximab plus chemotherapy. Br J Cancer 2007; 96: 1166-9.

11. De Roock W, Piessevaux H, De Schutter J, et al. KRAS wildtype state predicts survival and is associated to early radiological response in metastatic colorectal cancer treated with cetuximab. Ann Oncol 2008; 19: 508-15.

12. Karapetis CS, Khambata-Ford S, Jonker DJ, et al. K-ras mutations and benefit from cetuximab in advanced colorectal cancer. N Engl J Med 2008; 359: 1757-65.

13. Lievre A, Bachet JB, Boige $V$, et al. KRAS mutations as an independent prognostic factor in patients with advanced colorectal cancer treated with cetuximab. J Clin Oncol 2008; 26: 374-9.

14. Freeman DJ, Juan T, Reiner M, et al. Association of K-ras mutational status and clinical outcomes in patients with metastatic colorectal cancer receiving panitumumab alone. Clin Colorectal Cancer 2008; 7: 184-90.

15. Amado RG, Wolf $M$, Peeters $M$, et al. Wild-type KRAS is required for panitumumab efficacy in patients with metastatic colorectal cancer. J Clin Oncol 2008; 26: 1626-34.

16. Perkins G, Lievre A, Ramacci C, et al. Additional value of EGFR downstream signaling phosphoprotein expression to KRAS status for response to anti-EGFR antibodies in colorectal cancer. Int J Cancer 2010; 127: 1321-31.

17. Lievre A, Bachet JB, Le Corre D, et al. KRAS mutation status is predictive of response to cetuximab therapy in colorectal cancer. Cancer Res 2006; 66: 3992-5.

18. Saridaki Z, Tzardi M, Papadaki C, et al. Impact of KRAS BRAF, PIK3CA mutations, PTEN, AREG, EREG expression and skin rash in $>/=2$ line cetuximab-based therapy of colorectal cancer patients. PLoS One 2011; 6: e15980.

19. Samuels Y, Diaz LA Jr, Schmidt-Kittler O, et al. Mutant PIK3CA promotes cell growth and invasion of human cancer cells. Cancer Cell 2005; 7: 561-73.

20. Samuels Y, Wang Z, Bardelli A, et al. High frequency of mutations of the PIK3CA gene in human cancers. Science 2004; 304: 554.

21. Moroni M, Veronese S, Benvenuti S, et al. Gene copy number for epidermal growth factor receptor (EGFR) and clinical response to antiEGFR treatment in colorectal cancer: a cohort study. Lancet Oncol 2005; 6: 279-86.

22. Sartore-Bianchi A, Martini M, Molinari F, et al. PIK3CA mutations in colorectal cancer are associated with clinical resistance to EGFR-targeted monoclonal antibodies. Cancer Res 2009; 69: 1851-7.

23. Perrone F, Lampis A, Orsenigo $M$, et al. PI3KCA/PTEN deregulation contributes to impaired responses to cetuximab in metastatic colorectal cancer patients. Ann Oncol 2009; 20: 84-90.

24. Wong NS, Fernando NH, Nixon AB, et al. A phase II study of capecitabine, oxaliplatin, bevacizumab and cetuximab in the treatment of metastatic colorectal cancer. Anticancer Res 2011; 31: 255-61.
25. Prenen H, De Schutter J, Jacobs B, et al. PIK3CA mutations are not a major determinant of resistance to the epidermal growth factor receptor inhibitor cetuximab in metastatic colorectal cancer. Clin Cancer Res 2009; 15: 3184-8.

26. De Roock W, Claes B, Bernasconi D, et al. Effects of KRAS, BRAF, NRAS, and PIK3CA mutations on the efficacy of cetuximab plus chemotherapy in chemotherapy-refractory metastatic colorectal cancer: a retrospective consortium analysis. Lancet Oncol 2010; 11: 753-62.

27. Choi JH, Ahn MJ, Rhim HC, et al. Comparison of WHO and RECIST criteria for response in metastatic colorectal carcinoma. Cancer Res Treat 2005; 37: 290-3.

28. Lai YL, Mau BL, Cheng WH, Chen HM, Chiu HH, Tzen CY. PIK3CA exon 20 mutation is independently associated with a poor prognosis in breast cancer patients. Ann Surg Oncol 2008; 15: 1064-9.

29. Zhao L, Vogt PK. Helical domain and kinase domain mu tations in p110alpha of phosphatidylinositol 3-kinase induce gain of function by different mechanisms. Proc Natl Acad Sci U S A 2008; 105: 2652-7.

30. Kalinsky K, Jacks LM, Heguy A, et al. PIK3CA mutation associates with improved outcome in breast cancer. Clin Cancer Res 2009; 15: 5049-59.

31. Kim SY, Shim EK, Yeo HY, et al. KRAS mutation status and clinical outcome of preoperative chemoradiation with cetuximab in locally advanced rectal cancer: a pooled analysis of 2 phase II trials. Int J Radiat Oncol Biol Phys 2013; 85: 201-7.

32. Lin JK, Lin AJ, Lin CC, et al. The status of EGFR-associated genes could predict the outcome and tumor response of chemo-refractory metastatic colorectal patients using cetuximab and chemotherapy. J Surg Oncol 2011; 104: 661-6.

33. Cappuzzo F, Varella-Garcia M, Finocchiaro G, et al. Primary resistance to cetuximab therapy in EGFR FISH-positive colorectal cancer patients. Br J Cancer 2008; 99: 83-9.

34. Souglakos J, Philips J, Wang R, et al. Prognostic and predictive value of common mutations for treatment response and survival in patients with metastatic colorectal cancer. Br J Cancer 2009; 101: 465-72.

35. Tol J, Dijkstra JR, Klomp M, et al. Markers for EGFR pathway activation as predictor of outcome in metastatic colorectal cancer patients treated with or without cetuximab. Eur J Cancer 2010; 46: 1997-2009.

36. Sood A, McClain D, Maitra R, et al. PTEN gene expression and mutations in the PIK3CA gene as predictors of clinical benefit to anti-epidermal growth factor receptor antibody therapy in patients with KRAS wild-type metastatic colorectal cancer. Clin Colorectal Cancer 2012; 11 : 143-50.

37. Ulivi P, Capelli L, Valgiusti M, et al. Predictive role of multiple gene alterations in response to cetuximab in metastatic colorectal cancer: a single center study. J Transl Med 2012; 10: 87.

38. Mao C, Yang ZY, Hu XF, Chen Q, Tang JL. PIK3CA exon 20 mutations as a potential biomarker for resistance to anti-EGFR monoclonal antibodies in KRAS wild-type metastatic colorectal cancer: a systematic review and meta-analysis. Ann Oncol 2012; 23: 1518-25.

39. Spindler KL, Pallisgaard N, Lindebjerg J, Frifeldt SK, Jakobsen $A$. EGFR related mutational status and association to clinical outcome of third-line cetuximab-irinotecan in metastatic colorectal cancer. BMC Cancer 2011; 11: 107.

40. Soeda H, Shimodaira H, Watanabe M, et al. Clinical usefulness of KRAS, BRAF, and PIK3CA mutations as predictive markers of cetuximab efficacy in irinotecan- and 
oxaliplatin-refractory Japanese patients with metastatic colorectal cancer. Int J Clin Oncol 2013; 18: 670-7.

41. Molinari F, Felicioni L, Buscarino M, et al. Increased detection sensitivity for KRAS mutations enhances the prediction of anti-EGFR monoclonal antibody resistance in metastatic colorectal cancer. Clin Cancer Res 2011; 17: 4901-14.

42. Ikenoue T, Kanai F, Hikiba Y, et al. Functional analysis of PIK3CA gene mutations in human colorectal cancer. Cancer Res 2005; 65: 4562-7.

43. Markman B, Atzori F, Perez-Garcia J, Tabernero J, Baselga J. Status of PI3K inhibition and biomarker development in cancer therapeutics. Ann Oncol 2010; 21: 683-91.

44. Huang CH, Mandelker D, Schmidt-Kittler O, et al. The structure of a human p110alpha/p85alpha complex elucidates the effects of oncogenic PI3Kalpha mutations. Science 2007; 318: 1744-8.

45. Liao X, Morikawa T, Lochhead P, et al. Prognostic role of PIK3CA mutation in colorectal cancer: cohort study and literature review. Clin Cancer Res 2012; 18: 2257-68.

46. Farina-Sarasqueta A, van Lijnschoten G, Moerland E, et al. The BRAF V600E mutation is an independent prognostic factor for survival in stage II and stage III colon cancer patients. Ann Oncol 2010; 21: 2396-402.

47. Voutsina A, Tzardi M, Kalikaki A, et al. Combined analysis of KRAS and PIK3CA mutations, MET and PTEN expression in primary tumors and corresponding metastases in colorectal cancer. Mod Pathol 2013; 26: 302-13. 